INPLASY

PROTOCOL

To cite: Liu et al. The efficacy of hypoglycemic agents for alzheimer's disease and mild cognitive Impairment: A systematic review and metaanalysis. Inplasy protocol

202160075. doi:

10.37766/inplasy2021.6.0075

Received: 21 June 2021

Published: 21 June 2021

Corresponding author:

Qing Zhao

zhaoqing@jlu.edu.cn

Author Affiliation:

Department of Neurology,

China-Japan Union Hospital of

Jilin University.

Support: None.

Review Stage at time of this submission: The review has not yet started.

Conflicts of interest:

None declared.

\section{The efficacy of hypoglycemic agents for alzheimer's disease and mild cognitive Impairment: A systematic review and meta-analysis}

\author{
Liu, B1; Sun, W2; Zhao, Q³.
}

Review question / Objective: This review protocol aims to indicates whether anti-diabetic agents can serve as an effective and safe therapeutic for $A D$ and mild cognitive impairment patients.

Condition being studied: Numerous studies have demonstrated shared pathophysiological mechanisms between $A D$ and diabetes mellitus (DM) such as oxidative stress, mitochondrial dysfunction, inflammation, and insulin resistance. As the early stage of $A D, D M$ is not only a significant risk factor for mild cognitive impairment (MCl) but also shares several pathophysiological mechanisms, which is similar to AD. There are many ways to treat Alzheimer's disease, however, none of them can completely cure Alzheimer's dementia. Recently, more and more studies have demonstrated that hypoglycemic agents may have a benificial effect in Alzheimer's disease and $\mathrm{MCl}$. The main purpose of this review is to evaluate the effect of hypoglycemic agents on the cognitive function improvement of patients with Alzheimer's disease or $\mathrm{MCl}$.

INPLASY registration number: This protocol was registered with the International Platform of Registered Systematic Review and Meta-Analysis Protocols (INPLASY) on 21 June 2021 and was last updated on 23 September 2021 (registration number INPLASY202160075).

\section{INTRODUCTION}

Review question / Objective: This review protocol aims to indicates whether antidiabetic agents can serve as an effective and safe therapeutic for $A D$ and mild cognitive impairment patients.
Condition being studied: Numerous studies have demonstrated shared pathophysiological mechanisms between $A D$ and diabetes mellitus (DM) such as oxidative stress, mitochondrial dysfunction, inflammation, and insulin resistance. As the 
early stage of $A D, D M$ is not only a significant risk factor for mild cognitive impairment $(\mathrm{MCl})$ but also shares several pathophysiological mechanisms, which is similar to AD. There are many ways to treat Alzheimer's disease, however, none of them can completely cure Alzheimer's dementia. Recently, more and more studies have demonstrated that hypoglycemic agents may have a benificial effect in Alzheimer's disease and $\mathrm{MCl}$. The main purpose of this review is to evaluate the effect of hypoglycemic agents on the cognitive function improvement of patients with Alzheimer's disease or $\mathrm{MCl}$.

\section{METHODS}

Search strategy: We search the following databases in Chinese and English language before 1st August 2021 :China National Knowledge Infrastructure (CNKI), China Biological Medicine (CBM), Chinese Scientific Journals Database (VIP), Wanfang database, PubMed, EMBASE, and Cochrane Library. Chinese and American Clinical Trial Registration Center are also searched. Search keywords: "Alzheimer d i s e a s e ", "cog $n$ i $t$ i ve dysfunction", "hy pog l ycemic agents","metformin", "GLP-1", "Thiazolidi nediones", "insulin", "Sulfonylurea Compounds", "DDP-4", "a- Glucosidase inhibitors ", "Sodium-Glucose Transporter 2 Inhibitors".And research type includes: randomized controlled trial, controlled clinical trial, randomized.

Participant or population: Participants are diagnosed with Alzheimer's disease or MCl.

Intervention: Hypoglycemic agents.

Comparator: Participants without hypoglycemic agents therapy.

Study designs to be included: Randomized controlled trials.

Eligibility criteria: We will include randomized controlled trials (RCTs) study the effectiveness of hypoglycemic agents in the treatment of Alzheimer's disease.All the RCTs were published in English or Chinese, with the needed data for outcomes presented in a format which can be extracted for analysis.

Information sources: Nine databases of China National Knowledge Infrastructure(CNKI), WanFang Date, VIP, China Biology Medicine disc(CBM), PubMed, Embase, The Cochrane Library, Chinese and American Clinical Trial Registration Center are searched.

Main outcome(s): Changes from baseline in the cognition function of Alzheimer's disease with ADAS-cog or MMSE. Daily Living (ADL) scale is also taken into consideration.

Additional outcome(s): Cerebrospinal fluid amyloid beta and total tau concentration, adverse events are included. And HOMAIR, an index assessing insulin resistence also serves as an addition outcome.

Quality assessment / Risk of bias analysis: For assessing the methodological quality of included trials, we will use the Cochrane risk-of-bias tool, an instrument consisting of the following items: random sequence generation and allocation concealment (selection bias), blinding of participants and personnel (detection bias), blinding of outcome assessment(detection bias), incomplete outcome data (attrition bias), selective reporting of outcomes (reporting bias) and other biases (e.g., sponsorship bias). Each study will be given a quality rating such as "low risk of bias," "high risk of bias," or "unclear risk of bias" according to the presence of sufficient information and potential bias.

Strategy of data synthesis: We will used RevMan V.5.3 software for statistical analysis. For continuous variables, when outcomes were measured by the same scale, the results were reported as standardized mean difference(MD) and 95\% confidence interval( $\mathrm{Cl})$; when different scales were used, the results were reported as standardized mean difference 
(SMD) and 95\% Cl. Categorical data will be calculated with the risk ratio(RR) and $95 \%$ Cl. We will use $I^{2}$ test and $X^{2}$ test to evaluate the heterogeneity of the results. When $I^{2} \leq 50 \%$ or $P<0.10$, the results of the study will be considered as homogeneous, and fixed effect model will be used; otherwise, random effect model will be used.

Subgroup analysis: If there is significant heterogeneity in the included trials, and the necessary data are available, subgroup analyses will be done, according to subject characteristics (e.g., age, gender, and so on), subgroup analysis will be carried out according to the data retrieved.

Sensitivity analysis: Sensibility analysis will be applied if the heterogeneity is obvious.

Language: English and Chinese.

Country(ies) involved: China.

Keywords: hypoglycemic agents, alzheimer's disease, mild cognitive impairment, meta-analysis.

Contributions of each author:

Author 1 - Boyu Liu.

Author 2 - Wenyue Sun.

Author 3 - Qing Zhao. 